\title{
Novel 9q34.11 gene deletions encompassing combinations of four Mendelian disease genes: STXBP1, SPTAN1, ENG, and TOR1A
}

\author{
Ian M. Campbell, BS1, Svetlana A. Yatsenko, MD ${ }^{1,14}$, Patricia Hixson, PhD1', Tyler Reimschisel, MD², \\ Matthew Thomas, MS ${ }^{3}$, William Wilson, $\mathrm{MD}^{3}$, Usha Dayal, MD4, James W. Wheless, MD ${ }^{5}$, \\ Amy Crunk, $\mathrm{MS}^{6}$, Cynthia Curry, $\mathrm{MD}^{7}$, Nicole Parkinson, $\mathrm{MS}^{8}$, Leona Fishman, $\mathrm{MD}^{8}$, \\ James J. Riviello, MD ${ }^{9}$, Malgorzata J.M. Nowaczyk, MD ${ }^{10}$, Susan Zeesman, MS ${ }^{10}$, \\ Jill A. Rosenfeld, MS ${ }^{11}$, Bassem A. Bejjani, MD ${ }^{11}$, Lisa G. Shaffer, PhD ${ }^{11}$, \\ Sau Wai Cheung, PhD', James R. Lupski, MD, PhD ${ }^{1,12,13}$, \\ Pawel Stankiewicz, MD, PhD ${ }^{1}$ and Fernando Scaglia, MD ${ }^{1,13}$
}

\begin{abstract}
Purpose: A number of genes in the 9q34.11 region may be haploinsufficient. However, studies analyzing genotype-phenotype correlations of deletions encompassing multiple dosage-sensitive genes in the region are lacking.

Methods: We mapped breakpoints of 10 patients with 9q34.11 deletions using high-resolution 9q34-specific array comparative genomic hybridization $(\mathrm{CGH})$ to determine deletion size and gene content.

Results: The $9 \mathrm{q} 34.11$ deletions range in size from $67 \mathrm{~kb}$ to 2.8 $\mathrm{Mb}$. Six patients exhibit intellectual disability and share a common deleted region including $S T X B P 1$; four manifest variable epilepsy. In five subjects, deletions include SPTAN1, previously associated with early infantile epileptic encephalopathy, infantile spasms, intellectual disability, and hypomyelination. In four patients, the deletion includes endoglin $(E N G)$, causative of hereditary hemorrhagic telangiectasia. Finally, in four patients, deletions involve TOR1A,
\end{abstract}

of which molecular defects lead to early-onset primary dystonia. Ninety-four other RefSeq genes also map to the genomic intervals investigated.

Conclusion: STXBP1 haploinsufficiency results in progressive encephalopathy characterized by intellectual disability and may be accompanied by epilepsy, movement disorders, and autism. We propose that $9 \mathrm{q} 34.11$ genomic deletions involving ENG, TOR1A, STXBP1, and SPTAN1 are responsible for multisystemic vascular dysplasia, early-onset primary dystonia, epilepsy, and intellectual disability, therefore revealing cis-genetic effects leading to complex phenotypes.

Genet Med 2012:14(10):868-876

Key Words: cis-genetics, dystonia, early infantile epileptic encephalopathy, hereditary hemorrhagic telangiectasia, intellectual disability

\section{INTRODUCTION}

Neonatal or early infantile epileptic encephalopathies represent a class of severe epileptic disorders that can be caused by abnormal brain development as a result of congenital brain malformations or, less frequently, by inborn errors of metabolism. ${ }^{1}$ Early infantile epileptic encephalopathy (EIEE) with a burstsuppression pattern, also known by the eponym of Ohtahara syndrome, is a rare and severe form of epileptic encephalopathy characterized by early-onset of intractable seizures, with mostly tonic spasms or, more rarely, partial seizures and a specific pattern on electroencephalogram known as suppression burst. ${ }^{2}$ EIEE has a neonatal or early infantile onset and the natural course consists of progressive neurological dysfunction manifesting with concomitant cognitive and motor impairment leading to severe developmental delay (DD) or intellectual disability (ID). EIEE belongs to a broader class of disorders known as age-dependent epileptic encephalopathies, with evolving clinical phenotype and electroencephalogram characteristics as the brain matures. ${ }^{3}$ The majority of patients with EIEE subsequently develop infantile spasms (IS), or West syndrome, later in childhood. In some children, the epileptic phenotype may later evolve to Lennox-Gastaut syndrome by 6 years of age. ${ }^{4}$

The etiology of EIEE is heterogeneous; structural abnormalities of the brain such as Aicardi syndrome, cortical dysgenesis,

The first two authors contributed equally to this work.

B.A.B. is currently unaffiliated with Signature Genomic Laboratories.

${ }^{1}$ Department of Molecular and Human Genetics, Baylor College of Medicine, Houston, Texas, USA; ${ }^{2}$ Department of Pediatrics, Vanderbilt University Medical Center, Nashville,

Tennessee, USA; ${ }^{3}$ Department of Pediatrics, University of Virginia School of Medicine, Charlottesville, Virginia, USA; ${ }^{4}$ Carolina Neurological Clinic, Charlotte, North Carolina, USA; ${ }^{5}$ Department of Pediatric Neurology, University of Tennessee Health Science Center, Memphis, Tennessee, USA; ${ }^{6}$ Shodair Hospital, Helena, Montana, USA; ${ }^{7}$ Genetic Medicine of Central California, University of California San Francisco, Fresno, California, USA; ${ }^{8}$ Department of Pediatrics, Hospital for Sick Children, Toronto, Ontario, Canada; ${ }^{9}$ Department of Neurology, New York University Langone Medical Center, New York, New York, USA; ${ }^{10}$ Department of Pathology and Molecular Medicine and Pediatrics, McMaster University, Hamilton, Ontario, Canada; ${ }^{11}$ Signature Genomic Laboratories, PerkinElmer Inc., Spokane, Washington, USA; ${ }^{12}$ Department of Pediatrics, Baylor College of Medicine, Houston, Texas, USA; ${ }^{13}$ Texas Children's Hospital, Houston, Texas, USA. ${ }^{14}$ Present address: Obstetrics and Gynecology and Reproductive Sciences, Magee Women's Hospital, University of Pittsburgh School of Medicine, Pittsburgh, Pennsylvania, USA. Correspondence: Fernando Scaglia fscaglia@bcm.edu

Submitted 3 January 2012; accepted 9 May 2012; advance online publication 21 June 2012. doi:0.1038/gim.2012.65 
porencenphaly, and hemimegalencephaly may be accompanied by EIEE, whereas inborn errors of metabolism such as glycine encephalopathy and Leigh syndrome have been associated with other cases. ${ }^{2,4}$ More recently, studies have ascertained that EIEE with a burst-suppression pattern is genetically heterogeneous as well, with causative mutations identified in at least 12 genes (Supplementary Table S1, online)., ${ }^{1,5-8}$ In addition to the 12 known genes, one individual with EIEE and a de novo balanced translocation disrupting the SRGAP2 gene has recently been reported. ${ }^{9}$

Of note, two of the genes previously implicated in the etiology of EIEE, STXBP1, and SPTAN1, are both located within the same 9q34.11 genomic region. To date, only three patients with microdeletions encompassing both STXBP1 and SPTAN1 have been described. ${ }^{8,10,11}$ Two subjects presented with the characteristic clinical features of EIEE. ${ }^{8,11}$ Although the authors of the second study do not specifically mention SPTAN1, it and STXBP1 are likely deleted from the chromosome of their patient presenting with IS. ${ }^{10}$ De novo missense, nonsense, frameshift, and splice-site mutations, as well as genomic deletions of STXBP1 have been found in association with EIEE, suggesting STXBP1 haploinsufficiency as a cause of this epileptic phenotype. ${ }^{8,11-14}$ However, a 4-bp deletion within STXBP1 exon 4, resulting in a premature stop 41 codons downstream in the sixth of 20 exons, has been identified in one child of Japanese ancestry who presented with IS but did not exhibit the classical features consistent with EIEE. ${ }^{12}$ Furthermore, other publications reporting the clinical cases of patients of diverse ethnic backgrounds affected with epilepsy and carrying STXBP1 mutations sometimes failed to identify the burst-suppression pattern on electroencephalogram characteristic of EIEE. ${ }^{10,15,16}$ Finally, a de novo truncating STXBP1 mutation has been found in a 21-year-old patient with ID and no evidence of epilepsy, expanding the clinical spectrum associated with molecular defects of STXBP $1 .{ }^{17}$

Therefore, the complete range of phenotypic features caused by STXBP1 mutations and deletions remains to be elucidated. Herein, we present clinical and high-resolution molecular studies of 10 patients with 9q34.11 deletions in order to better delineate the clinical manifestations associated with STXBP1 and SPTAN1 haploinsufficiencies and to characterize the clinical presentations associated with possible cis-genetic effects when additional potentially dosage sensitive genes are involved.

\section{MATERIALS AND METHODS}

\section{Human subjects}

All human subjects research was approved by the Institutional Review Board of Baylor College of Medicine (BCM). We studied 10 unrelated children (six males and four females, patient (P)1-P10) with variable clinical phenotypes, who were found to have a loss in DNA copy number in the $9 \mathrm{q} 34.11$ region revealed by array comparative genomic hybridization (CGH). Each subject was originally identified among patients referred for clinical array $\mathrm{CGH}$ at the BCM Medical Genetics Laboratory (P3-P6) or PerkinElmer
Signature Genomic Laboratories (P1, P2, and P7-P10) for a diverse range of clinical indications. Four patients (P1, P2, P7, and P8) were identified by SignatureChipOS v1.1 105K oligo whole-genome array (Agilent Technologies, Santa Clara, CA) and two patients (P9 and P10) were identified by SignatureChipOS v2.0 135K oligo whole-genome array (Roche NimbleGen, Madison, WI). P4 was identified by BCM CMA v7.1 $105 \mathrm{~K}$ oligo whole-genome array (Agilent Technologies). P6 was identified by BCM CMA v7.4 105K oligo exon-targeted array (Agilent Technologies). P3 and P5 were identified by BCM CMA v8.1 180K oligo exontargeted array (Agilent Technologies). Informed consent for the research use of genetic material and clinical information was obtained from the parent or guardian of each patient. Peripheral blood samples were obtained from individuals and available family members. All subjects had apparently normal G-banded chromosome analyses. Clinical details appear in Supplementary Materials and Methods, online.

\section{Array-CGH studies}

Patients' genomic DNA was extracted from peripheral blood samples according to the manufacturers' protocols. Two separate custom, high-resolution 9q34 arrays were designed using a $60 \mathrm{~K}$ array format (Agilent Technologies, Santa Clara, CA) and were used on the basis of availability at time of patient ascertainment. Version 1 of the custom array had an average probe spacing of $\sim 1,250$ bp per probe; version 2 had an average spacing of $\sim 250 \mathrm{bp}$ per probe. Sex-matched DNA samples from either a normal male or female individual were used as a hybridization reference control. DNA digestion, labeling, and hybridization were performed according to the manufacturer's instructions (Agilent Technologies). Data were analyzed using Agilent Genomic Workbench Software (Agilent Technologies) and plotted using the R Statistical Computing Package (R Core Development Team). Insufficient DNA was available for direct analysis of $\mathrm{P} 4$ and P8; therefore, whole-genome amplification was performed on patient and control DNA according to the manufacturer's instructions (Sigma-Aldrich, St Louis, MO) before array hybridization.

\section{Fluorescence in situ hybridization analysis}

Chromosomes for fluorescence in situ hybridization analyses were obtained from peripheral blood lymphocytes using standard procedures to independently confirm the findings detected by array CGH. Fluorescence in situ hybridization studies were performed on all children and available parents using bacterial artificial chromosome or fosmid probes mapped within the $9 \mathrm{q} 34.11$ region.

\section{Polymerase chain reaction breakpoint analysis}

Polymerase chain reaction (PCR) primers were designed to flank each patient's deletion breakpoints on the basis of aCGH data. Long-range PCR was employed to amplify the deletion-specific junction fragment using the manufacturer's protocol (Takara Bio, Otsu, Japan). PCR products were Sanger sequenced (Lone 
Star, Houston, TX) and compared with the reference human genome sequence using the UCSC Genome Browser.

\section{RESULTS}

We identified 10 patients with microdeletions involving the 9q34.11 region among patients referred for array CGH for a diverse set of clinical indications at two diagnostic laboratories. All deletions were also observed with fluorescence in situ hybridization analysis using bacterial artificial chromosome or fosmid probes specific for the deleted region as an independent experimental verification. Clinical manifestations are summarized in Table 1. The deletions were de novo in eight of eight cases in which parents were available.

To determine the size, extent, and genomic content of the deletions, patients' samples were analyzed by custom-designed, high-resolution 9q34 array CGH. The genomic intervals deleted and the corresponding nucleotide positions of the breakpoints (GRChr37/hg19, 2009) were delineated (Figure 1, Table 2). The deletions vary in size from $67 \mathrm{~kb}$ to $2.8 \mathrm{Mb}$ and encompass multiple genes (Figure 1, Supplementary Table S2, online). The exact locations and nucleotide sequences of breakpoint junctions were obtained for four selected subjects by Sanger sequencing (Supplementary Figure S1, online).

The $9 \mathrm{q} 34.11$ region includes at least four relevant disease genes, including STXBP1 (encoding syntaxin binding protein 1, MIM 602926), ENG (encoding endoglin, MIM 131195), SPTAN1 (encoding spectrin, alpha, non-erythrocytic 1, MIM 182810), and TOR1A (encoding torsin family 1, member A, MIM 605204); single nucleotide variants of each gene have been associated with distinct genetic conditions: early infantile epileptic encephalopathy type 4 (EIEE4), hereditary hemorrhagic telangiectasia type 1 (HHT1), early infantile epileptic encephalopathy type 5 (EIEE5), and torsion dystonia type 1 , respectively. In three patients (P1-P3), deletions involved the STXBP1, SPTAN1, and ENG genes (Figure 1, Table 1). In P1, the proximal deletion breakpoint was located within intron 15 of the $S T X B P 1$, removing the $3^{\prime}$ gene portion, including exons 16-20 from isoform A and exons 16-19 from isoform B (Figure 2). P1 was also the only member of our cohort whose deletion involves all four discussed genes (Figure 1). In P10, the 9q34.11 deletion encompassed two disease genes, STXBP1 and ENG (Figure 1). The smallest deletion, $67 \mathrm{~kb}$, involved exons 1-4 of the STXBP1 gene and was found in P5 (Figure 2). Deletions in two patients (P6 and P9) included SPTAN1 but did not involve STXBP1 (Figure 1). In P6 the TOR1A gene was also deleted (Figure 1). Deletions containing TOR1A were also found in P1, P7, and P8 (Figure 1). A total of five patients, P5 in our study, two patients reported by Deprez et al., ${ }^{16}$ one patient reported by Milh et al., ${ }^{14}$ and one patient reported by Saitsu et al., ${ }^{11}$ had small deletions including annotated coding sequence of only STXBP1 (Figure 2).

Although we did not observe any correlation between deletion size and the severity of patients' phenotypes, correlations between specific phenotypic features and gene content of a patient's copy-number variation were apparent (Table 1).
Subjects with deletions of STXBP1 coding sequence were more likely to have epilepsy. Moreover, one patient with disruption of SPTAN1 displayed defects in myelination. One individual with a deletion encompassing the TOR1A gene manifested dystonia. Finally, a patient with an $E N G$ deletion was found to have an arteriovenous malformation.

\section{DISCUSSION}

Deletions involving 9q34.11 result in a wide range of clinical phenotypes because of differences in the deletion size, gene content within the deleted region, and function of each individual affected gene. There are at least four known genes mapped to this region that are apparently dosage sensitive, STXBP1, SPTAN1, ENG, and TOR1A. Each individual gene, and potentially selected combinations in cis, may contribute to the complex phenotypes observed in patients with 9q34.11 deletions.

STXBP1 encodes syntaxin binding protein 1, an evolutionarily conserved neuronal protein that is vital for the process of calcium ion-dependent exocytosis in neurons as well as in neuroendocrine cells. ${ }^{18,19}$ STXBP1, a member of the SM (Sec1/ Munc-18) family, binds to syntaxin, thus promoting its stability, and regulates soluble $\mathrm{N}$-ethylmaleimide-sensitive factor attachment protein receptor (SNARE) complex formation with ensuing priming and fusion of synaptic vesicles. Therefore, STXBP1 has a fundamental function in synaptic vesicle release, acting in conjunction with the vesicular SNARE protein vesicle-associated membrane protein 2 (VAMP2, synaptobrevin 2) and the target membrane SNARE proteins syntaxin 1 and synaptosomal-associated protein 25 (SNAP25) to facilitate membrane fusion and neurotransmitter release. ${ }^{19,20}$

Patients with EIEE4 have been found to have missense, nonsense, frameshift, and splice-site mutations as well as wholegene and intragenic deletions of STXBP1., ${ }^{8,13,14,21}$ In addition, there is increasing awareness that $S T X B P 1$ mutations may also cause IS as the presenting epileptic feature, broadening the recognized clinical phenotypic consequences of genetic variation at the STXBP1 locus. ${ }^{10,12,16}$ In one of the same studies implicating a link between STXBP1 and IS, five of the six subjects harboring STXBP1 mutations manifested nonspecific epileptic phenotypes. ${ }^{16}$ Similarly, in another report, two individuals with STXBP1 mutations were affected with nonsyndromic epilepsy. ${ }^{15}$ In our cohort, only four of six subjects (P2-P5 Table 1) identified with STXBP1 coding sequence deletions presented with epilepsy. Each patient's constellation of findings lacked the clinical and electroencephalogram characteristics suggestive of EIEE, supporting the concept that alterations in STXBP1 coding sequence may be associated with diverse epileptic presentations. Our patients' phenotypes were consistent with IS with later evolution to infantile myoclonic epilepsy (P2), tonic localization related epilepsy (6 years, P3), nocturnal myotonic atonic epilepsy (P4), and neonatal-onset generalized epilepsy with later evolution to Lennox-Gastaut syndrome (P5). Of note, one patient with a partial STXBP1 deletion (patient P5) exhibited a severe neurological phenotype as did three previously reported patients with intragenic STXBP1 deletions (Figure 2), 


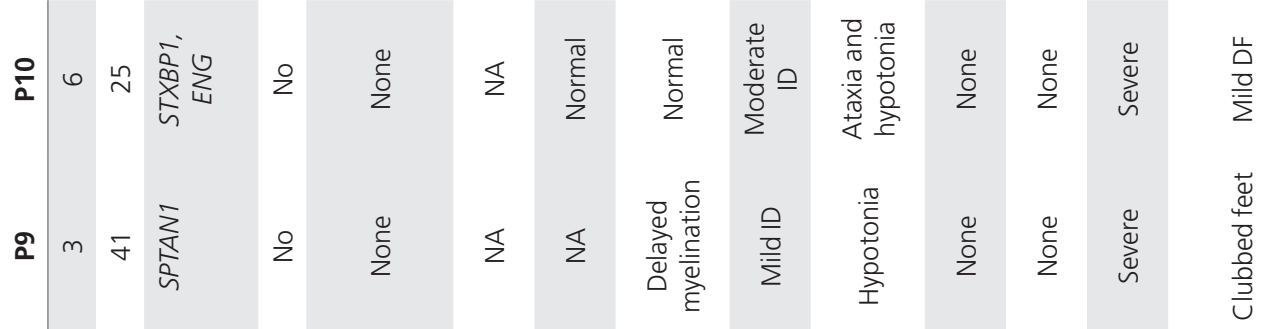

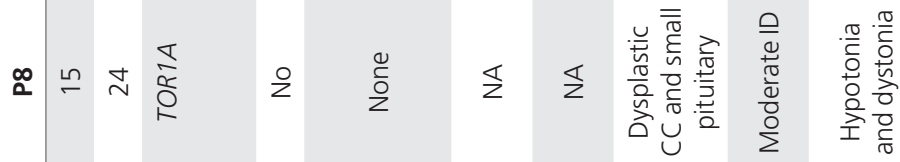

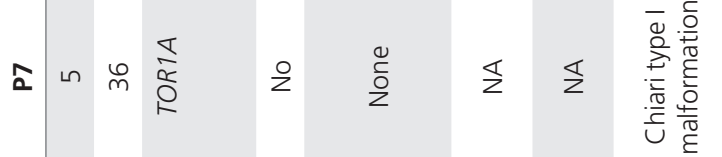

$\frac{0}{y}$
$\frac{\pi}{\pi}$
$\frac{\pi}{2}$
$\frac{0}{2}$

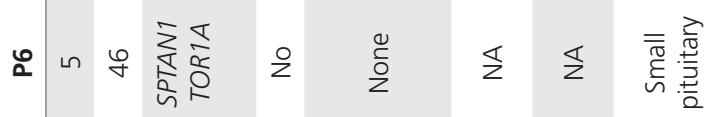

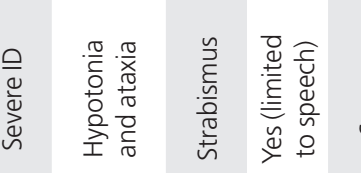

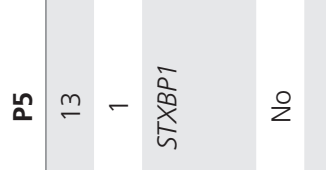

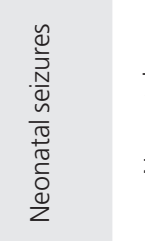

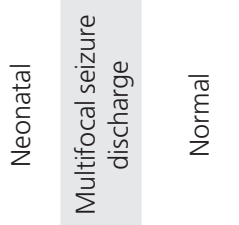

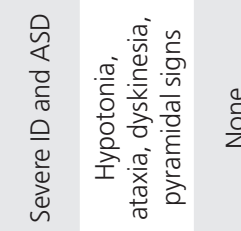

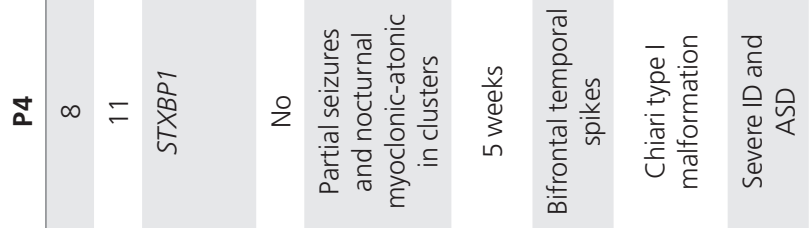

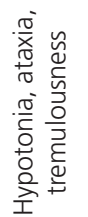

$\stackrel{\check{c}}{\frac{0}{+}}$

$\sqrt{n+2}$

$\frac{0}{0} \stackrel{0}{\circ}$

$\frac{1}{\sqrt{2}}$

बृ

孚

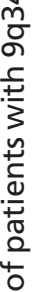

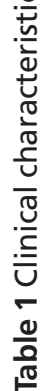

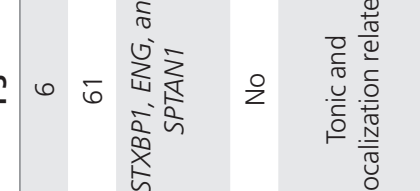

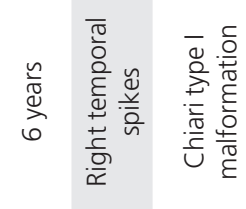

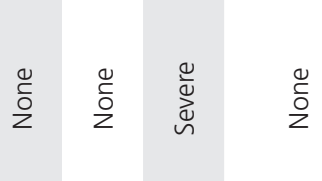

$\pi\left(\frac{1}{2}\right.$

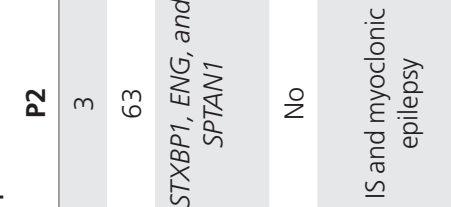

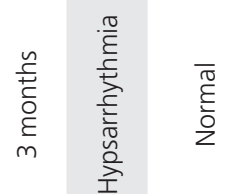

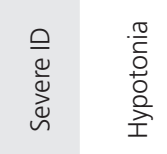

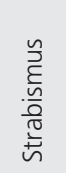

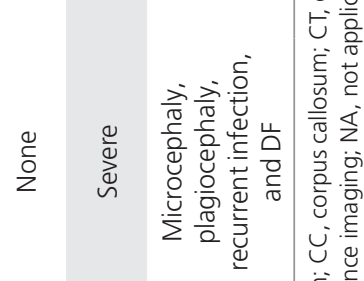

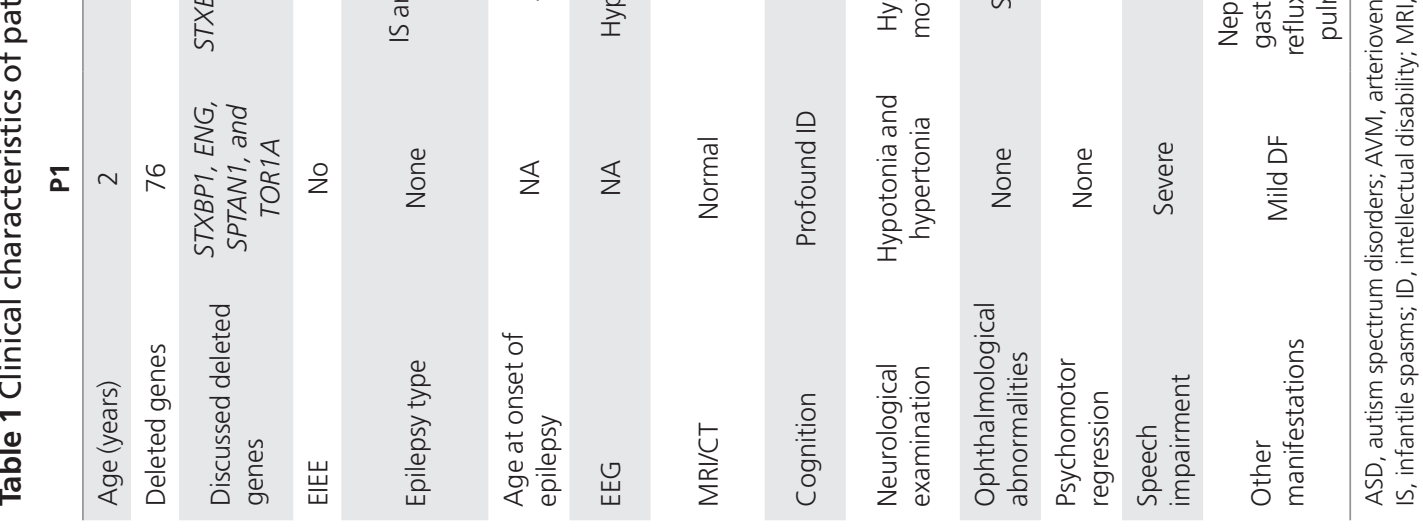




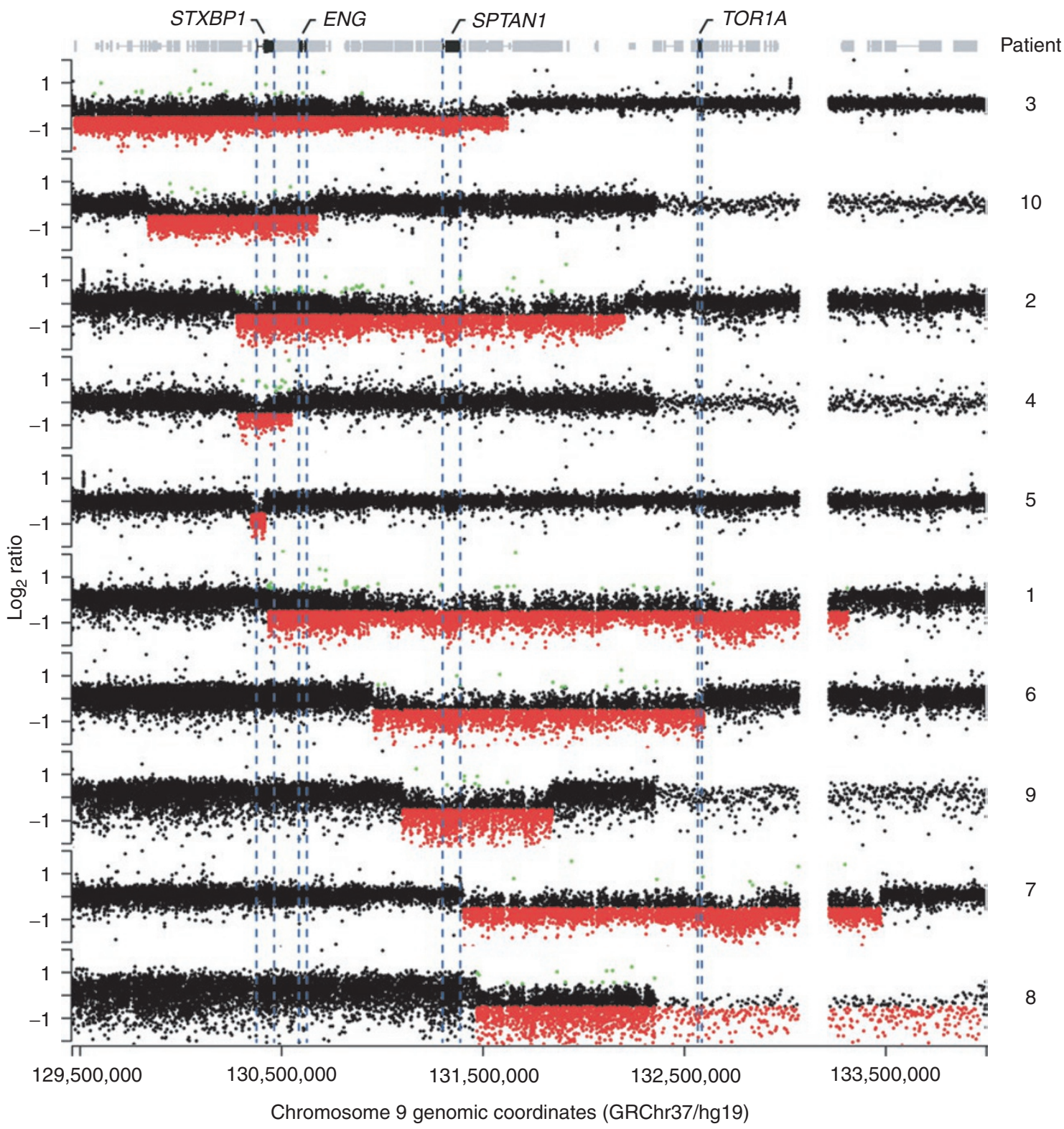

Figure 1 Custom-designed high-resolution array comparative genomic hybridization (CGH) analysis of the $9 q 34.11$ region, including genes of interest (in scale). Array CGH probes with absolute value $\log _{2}$ patient:control ratios greater than 0.5 within the deleted region are colored for ease of visualization. The locations of the STXBP1, ENG, SPTAN1, and TOR1A genes are indicated along the top of the graph in black. Ninety-four additional RefSeq genes are depicted as horizontal gray rectangles. All data are presented according to the GRChr37/hg19 assembly.

suggesting that isolated STXBP1 haploinsufficiency is associated with epilepsy and progressive neurodegeneration.

Response to antiepileptic therapy in our cohort varied extensively; three patients (P3-P5) exhibited intractable epilepsy, whereas another (P2) responded to treatment at 8 months of age. In three other studies, some patients demonstrated a beneficial response to treatment with vigabatrin alone or in combination with other antiepileptic compounds and continued clinical resolution of early epileptic features. ${ }^{10,14,16}$ Nevertheless, the number of patients evaluated is small and the natural history associated with STXBP1 alterations remains to be delineated.
Therefore, robust conclusions regarding therapeutic approaches or their efficacy remain undefined.

Two patients in our cohort harboring STXBP1 deletions (P1 and P10; Table 1) had no evidence of epilepsy at age 2 or 6 years, respectively, and presented with phenotypes consistent with severe to profound nonsyndromic ID. These two patients, together with a 21-year-old subject with a de novo truncating STXBP1 mutation (Supplementary Table S3, online) and no epilepsy reported by Hamdan et al., ${ }^{17}$ suggest that STXBP1 alterations may lead to a phenotype of severe to profound ID without overt epilepsy or abnormal 
Table 2 Summary of array-CGH results in patients with $9 q 34.11$ deletions

\begin{tabular}{|c|c|c|c|c|}
\hline Patient & Sex & Deletion size (Mb) & RefSeq genes involved ${ }^{a}$ & Genomic location (GRCh37/hg19) \\
\hline P1 & Female & 2.878 & $\begin{array}{l}\text { STXBP1 (exons 16-20), ENG, SPTAN1, TOR1A, } \\
\text { and } 72 \text { others }\end{array}$ & chr9:130439828-133320690 \\
\hline P2 & Female & 1.765 & STXBP1, ENG, SPTAN1, and 60 others & chr9:130281799-132208337 \\
\hline P3 & Male & 2.649 & STXBP1, ENG, SPTAN1, and 58 others & chr9:129473714-131633299 \\
\hline P4 & Male & 0.251 & STXBP1 and 10 others & chr9:130291275-130554161 b \\
\hline P5 & Female & 0.067 & STXBP1 (exons 1-4) & chr9:130353624-130421329b \\
\hline P7 & Male & 1.967 & TOR1A and 35 others & chr9:131406317-133482292 \\
\hline P8 & Male & 2.349 & TOR1A and 23 others & chr9:131472698-133975666 \\
\hline P9 & Male & 0.713 & SPTAN 1 and 40 others & chr9:131103396-131850003 \\
\hline P10 & Male & 0.808 & STXBP1, ENG, and 23 others & chr9:129843508-130681956b \\
\hline
\end{tabular}

aFor a complete list of RefSeq genes involved in each patient's deletion, see Supplementary Table S2 online.

bExact coordinates confirmed by Sanger sequencing (see Supplementary Figure S1 online).

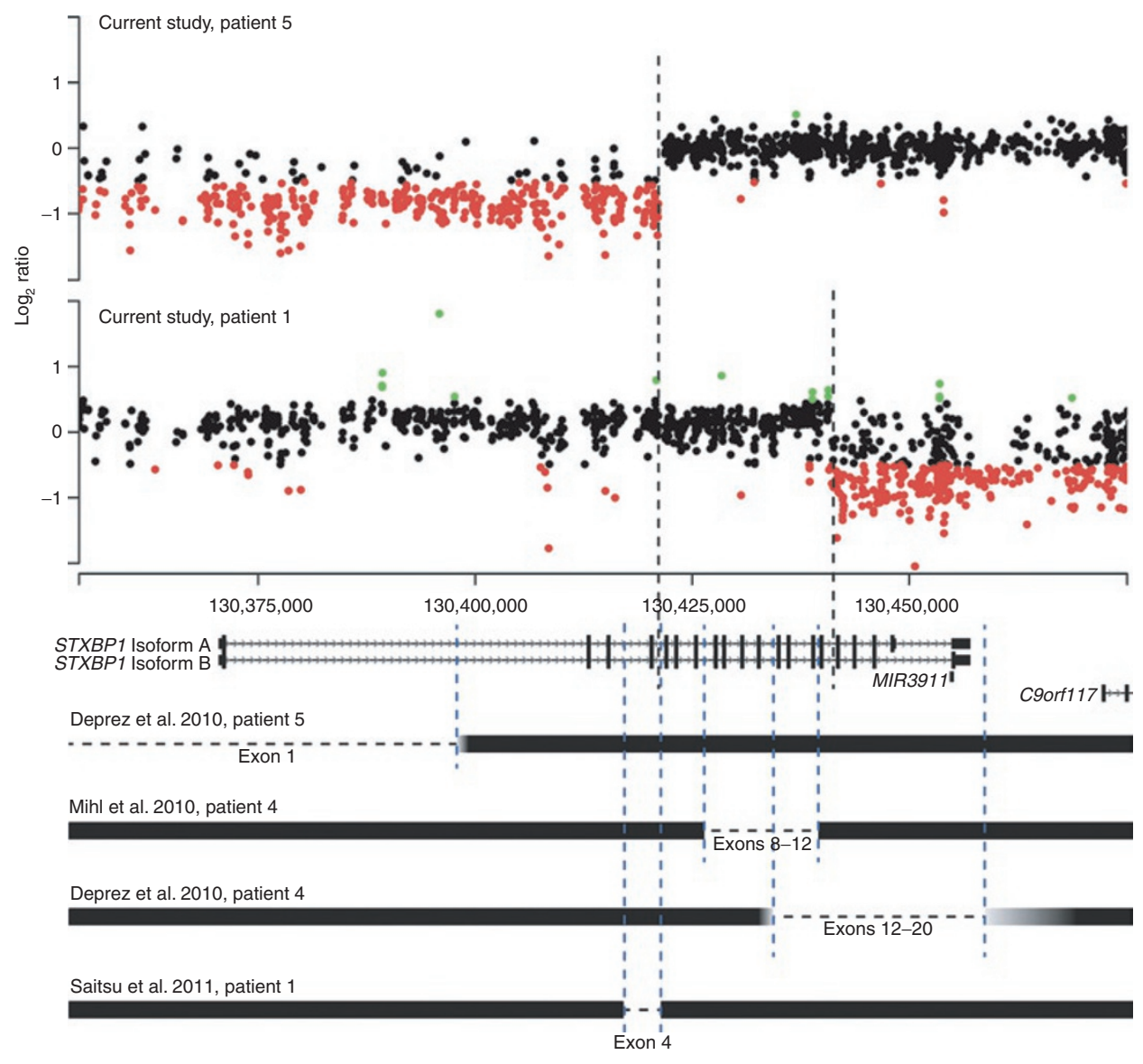

Figure 2 Single gene deletions of STXBP1 coding sequence. Top, array comparative genomic hybridization analysis of the STXBP1 coding region in two patients from this study with partial deletions of STXBP1. Middle, schematic representation of the two RefSeq isoforms of STXBP1. Below, schematic representation of the deletions involving segments of the STXBP1 gene in four previously reported patients. Dashed lines represent the extent of the genomic deletion in the previous patients. Black horizontal bars represent nondeleted regions; gradient bars identify either uninformative or inconclusive areas.

electroencephalogram. Furthermore, the severe encephalopathy associated with STXBP1 mutations may be independent of the presence or severity of epilepsy. However, because P1 is younger than the oldest age at epilepsy presentation in our cohort (P3) and P10 is the same age, it is possible that one or both could experience more seizures as their brains mature.

Symptoms consistent with autism spectrum disorders (ASDs) were found in two patients (P4 and P5, Table 1) with 
STXBP1 deletions. These findings are not surprising given the high comorbidity of ID and ASDs and the recent work delineating mutations in synaptogenesis and synaptic function genes in association with ASDs. ${ }^{22-24}$ Whereas a previous study did not identify STXBP1 mutations or deletions in 142 subjects with ASDs,${ }^{15}$ these results should be interpreted with caution, as the criteria used to diagnose ASDs in that study were not reported and the cohort was too small to evaluate for potential rare variants. ${ }^{25}$ In contrast, a more recent study demonstrated autistic features in three subjects with STXBP1 mutations, ${ }^{14}$ suggesting that ASDs may be part of the broad clinical spectrum associated with STXBP1 disruption.

Additional neurological features, including motor dyspraxia, ataxia, tremor, dyskinesia, and pyramidal signs, were observed in association with STXBP1 deletions (in P2, P4-P6, and P10, Table 1). Literature review reveals detection of several types of nonepileptic neurological features in subjects carrying singlenucleotide variant and copy-number variation mutations of STXBP1. Diffuse tremor was observed in two cases reported by Hamdan et al., ${ }^{17}$ three cases reported by Mignot et al., ${ }^{10}$ and two cases described by Milh et al. ${ }^{14}$ Ataxia and dyskinesia were observed in five subjects studied by Deprez et al., ${ }^{16}$ and myoclonic jerks were reported in four cases described by Milh et al. ${ }^{14}$ Stereotypic hand movements were noticed in the three subjects reported by Mignot et al..$^{10}$ and in two subjects reported by Milh et al. ${ }^{14}$ Moreover, a number of subjects harboring a missense mutation in STXBP1 and exhibiting nonepileptic movement symptoms have been described (Supplementary Table S3, online) ${ }^{8,14,26,27}$ In the context of neurophysiological consequences of STXBP1 variation, many of these neurological manifestations may be the result of impaired release of excitatory and inhibitory neurotransmitters that normally regulate the function of the indirect pathway of the motor loop. This circuit connects the basal ganglia, thalamus, and motor cortex, and its disruption may lead to involuntary movement activity. ${ }^{28}$ These associated neurological findings suggest that early disruption in brain development and maturation of the central nervous system caused by STXBP1 mutations and deletions may result in a broad constellation of clinical neurological findings, including different epilepsies, isolated nonsyndromic ID, movement disorders, and ASDs with the ensuing phenotype depending on the additive effects of genetic modifiers and environmental factors for each affected individual. ${ }^{25}$

Deletions in five subjects in our cohort (P1-P3, P6, and P9; Table 1) involve the entire SPTAN1 coding sequence. Recent reports have shown that alterations in SPTAN1 may present with IS, severe central hypomyelination, and ID. ${ }^{29}$ SPTAN1 encodes $\alpha-2$ spectrin, the major $\alpha$-spectrin expressed in non-erythroid cells and an essential protein involved in proper myelination in zebrafish. ${ }^{30}$ Two subjects with SPTAN1 mutations and one with a complex rearrangement of $9 \mathrm{q} 34.11$ involving both STXBP1 and SPTAN1 presented with early-onset IS, severe hypomyelination, spastic quadriplegia, visual impairment, and global DD. ${ }^{29}$ Moreover, a separate group has reported another subject with deletion of both STXBP1 and SPTAN1 who presented with
IS, generalized tremor, stereotypic movements, global cerebral atrophy, and ID. ${ }^{14}$

Three patients reported here have 9q34.11 deletions encompassing both STXBP1 and SPTAN1 (P1-P3; Figure 1, Table 1), and two patients have deletions of SPTAN1 without deletion of STXBP1 coding sequence (P6 and P9; Figure 1, Table 1). Nonetheless, only two patients (P2 and P3) in our cohort manifested epilepsy, and both of these patients' deletions also harbored STXBP1 coding sequence. These results suggest that SPTAN1 haploinsufficiency alone may not necessarily lead to an epileptic phenotype. Moreover, although hypomyelination and atrophy of the brainstem and cerebellum were reported before in patients with SPTAN1 mutations, only P9 from this study had documented hypomyelination on neuroimaging. These findings point towards a less deleterious effect associated with loss-of-function mutations as compared to a potential dominant negative (antemorphic) or gain-of-function (neomorphic) effect caused by in-frame SPTAN1 mutations. It has been postulated that in-frame SPTAN1 mutations may exert a dominant negative effect by aggregation of mutant $\alpha$-II/ wild-type $\beta$-II and mutant $\alpha$-II/wild-type $\beta$-III spectrin heterodimers. ${ }^{29}$ Such defective heterodimers may cause instability of membrane proteins, including ion channels, localized to the axon initial segment, while simultaneously altering intracellular vesicular transport. ${ }^{31}$ Therefore, distorted cytoskeletal scaffolds caused by the effect of in-frame SPTAN1 mutations may result in more detrimental neurological phenotypes. ${ }^{29}$ These findings could explain the absence of epilepsy in some patients with SPTAN1 deletions described in this study and the finding of hypomyelination in only one of the subjects reported herein. When hypomyelination was previously reported in subjects with SPTAN1 deletion, a normal myelination pattern was observed over time. ${ }^{29}$

Genomic deletions involving ENG have been observed in individuals with hereditary hemorrhagic telangiectasia type 1 (HHT1), ${ }^{32,33}$ supporting the hypothesis that ENG haploinsufficiency is the molecular etiology of HHT1. ENG disruption leads to abnormal vascular development ranging from dilated microvessels to large arteriovenous malformations with potentially lethal complications. HHT1-associated phenotypes can be quite variable, but the disorder often presents with early-onset recurrent spontaneous epistaxis. Four patients (P1-P3 and P10; Table 1) in this study have deletions involving the ENG gene. In patient 2, a pulmonary arteriovenous malformation was discovered incidentally upon evaluation for respiratory distress and low oxygen saturation levels. As the clinical phenotype associated with HHT1 tends to develop during childhood and adolescence and paucity of clinical features such as epistaxis, telangiectasias, or symptoms of solid organ arteriovenous malformation are not uncommon in affected children, ${ }^{34}$ it is not surprising to find that the other three patients harboring $E N G$ deletions in our cohort do not manifest symptoms. Clinical findings in P2 illustrate the necessity of high-resolution molecular analysis to precisely identify gene content in patients with $9 \mathrm{q} 34.11$ deletions, which can be used for accurate genetic 
counseling and appropriate monitoring for early detection of systemic complications when $E N G$ is deleted.

In four members of our cohort (P1 and P6-P8; Table 1) the TOR1A gene is deleted. TOR1A encodes the protein torsin-1A (torsin A). A 3 bp deletion, c.907_909delGAG, in this gene has been found in all patients with early-onset primary torsion dystonia type 1 in whom a definitively causative molecular lesion has been identified..$^{35}$ The disease is more prevalent in patients of Ashkenazi Jewish ancestry because of a founder effect. ${ }^{36}$ Torsion dystonia type 1 is an autosomal dominant condition with agedependent penetrance; asymptomatic subjects known to harbor the TOR1A disease-causing mutation have a $30-40 \%$ chance of developing clinical manifestations. Evidence from mouse models suggests TOR1A c.907_909delGAG is a loss-of-function allele, but a dominant negative (antemorphic) effect cannot be excluded. ${ }^{37}$ Decreased penetrance may be because of the presence of an in trans genetic modifier within the TOR1A gene itself..$^{38}$ Thus, the fact that only one (P8) of our four patients with TOR1A deletions exhibits dystonia is not unexpected. Aside from the manifestation of dystonia and tremor, patients with torsion dystonia type 1 display no other neurological or cognitive abnormalities. Therefore, the presence of moderate ID and dysmorphic features in the four subjects of our cohort is probably because of the influence of deleted neighboring genes.

In addition to the four genes detailed above, coding sequence of 94 other RefSeq genes was contained within the deletion of at least one patient in our cohort (Supplementary Table S2, online). None of the additional genes can be found in a previously published list of haploinsufficient genes. ${ }^{39}$ Nine of these genes are associated with human phenotypes: SLC27A4 (MIM 608649), AK1 (MIM 612631), LAMC3 (MIM 614115), DOLK (MIM 610768), LRRC8A (MIM 613506), LRSAM1 (MIM 614436), GLE1 (MIMs 253310, 611890), ASS1 (MIM 215700), and MIR2861 (MIM 613418). However, among all of the phenotypes listed, only the underlying molecular mechanism for Charcot-Marie-Tooth type 2P, caused by defects in LRSAM1, may be possibly related to haploinsufficiency. ${ }^{40}$ Because the earliest onset of CMT type $2 \mathrm{P}$ is during the third decade of life, haploinsufficiency of LRSAM1 is unlikely to contribute to the current phenotypes of $\mathrm{P} 3$ and $\mathrm{P} 10$.

Overall, seven of the 10 patients had mildly dysmorphic features; however, these features were heterogeneous, and no characteristic facial "gestalt" is shared among them. A longitudinal clinical study enrolling a larger number of subjects with these deletions will be required to identify potentially consistent dysmorphic features and to delineate the extent to which dosagesensitive gene content could play a role.

In conclusion, interstitial nonrecurrent deletions in the gene-rich 9q34.11 region in our patients vary in size from 67 $\mathrm{kb}$ to $2.8 \mathrm{Mb}$ and have different breakpoints. The microdeletions often span multiple dosage-sensitive genes, suggesting that the resultant overall phenotype could arise from combinations of cis-genetic effects. Physical proximity of at least four dosage-sensitive genes (STXBP1, SPTAN1, ENG, and TOR1A) within the $9 \mathrm{q} 34.11$ region adds further complexity to the observed phenotype and clinical course of these subjects. We propose that high-resolution chromosomal microarray analysis, including coverage for $9 \mathrm{q} 34.11$ microdeletions, should be considered in infants and children with early-onset epilepsy or severe progressive encephalopathy associated with DD or ID; STXBP1 haploinsufficiency may be an under-ascertained cause of such phenotypes. Patients with 9q34.11 deletions involving the $E N G$ gene can present with arteriovenous malformations in lungs, spinal cord, and brain; those with deletions involving the TOR1A gene can present with dystonia. The identification and extensive genomic and clinical studies of additional patients is necessary to elucidate the role of SPTAN1 haploinsufficiency as well as the clinical consequences of other dosage-sensitive genes and cis-genetic effects in the 9q34.11 region.

\section{SUPPLEMENTARY MATERIAL}

Supplementary material is linked to the online version of the paper at http://www.nature.com/gim

\section{ACKNOWLEDGMENTS}

We thank the families for their cooperation. This study was supported in part by grants from the Intellectual and Developmental Disabilities Research Center (P30 HD024064) and the National Institute of Neurological Disorders and Stroke (R01 NS058529) to J.R.L.

\section{DISCLOSURE}

J.R.L. is a consultant for Athena Diagnostics, 23andMe, lon Torrent Systems Inc., and holds multiple US and European patents for DNA diagnostics. Furthermore, the Department of Molecular and Human Genetics at Baylor College of Medicine derives revenue from molecular diagnostic testing (http://www.bcm.edu/geneticlabs/). J.A.R. and L.G.S. are employees of Signature Genomics, a subsidiary of PerkinElmer, Inc. The other authors declare no conflict of interest.

\section{REFERENCES}

1. Molinari F, Raas-Rothschild A, Rio M, et al. Impaired mitochondrial glutamate transport in autosomal recessive neonatal myoclonic epilepsy. Am J Hum Genet 2005;76:334-339

2. Ohtahara S, Ohtsuka Y, Yamatogi Y, Oka E. The early-infantile epileptic encephalopathy with suppression-burst: developmental aspects. Brain Dev 1987;9:371-376

3. Zupanc ML. Clinical evaluation and diagnosis of severe epilepsy syndromes of early childhood. J Child Neuro/ 2009;24(8 Suppl):6S-14S

4. Ohtahara S, Yamatogi Y. Epileptic encephalopathies in early infancy with suppression-burst. J Clin Neurophysio/ 2003;20:398-407.

5. Strømme P, Mangelsdorf ME, Shaw MA, et al. Mutations in the human ortholog of Aristaless cause X-linked mental retardation and epilepsy. Nat Genet 2002;30:441-445

6. Weaving LS, Christodoulou J, Williamson SL, et al. Mutations of CDKL5 cause a severe neurodevelopmental disorder with infantile spasms and mental retardation. Am J Hum Genet 2004;75:1079-1093.

7. Kato M, Saitoh S, Kamei A, et al. A longer polyalanine expansion mutation in the ARX gene causes early infantile epileptic encephalopathy with suppression-burst pattern (Ohtahara syndrome). Am J Hum Genet 2007;81:361-366.

8. Saitsu H, Kato M, Mizuguchi T, et al. De novo mutations in the gene encoding STXBP1 (MUNC18-1) cause early infantile epileptic encephalopathy. Nat Genet 2008;40:782-788.

9. Saitsu H, Osaka H, Sugiyama S, et al. Early infantile epileptic encephalopathy associated with the disrupted gene encoding Slit-Robo Rho GTPase activating protein 2 (SRGAP2). Am J Med Genet A 2011; e-pub ahead of print. 
10. Mignot C, Moutard ML, Trouillard O, et al. STXBP1-related encephalopathy presenting as infantile spasms and generalized tremor in three patients. Epilepsia 2011;52:1820-1827.

11. Saitsu $H$, Kato M, Shimono M, et al. Association of genomic deletions in the STXBP1 gene with Ohtahara syndrome. Clin Genet 2012;81:399-402.

12. Otsuka M, Oguni H, Liang JS, et al. STXBP1 mutations cause not only Ohtahara syndrome but also West syndrome-result of Japanese cohort study. Epilepsia 2010;51:2449-2452.

13. Saitsu H, Kato M, Okada I, et al. STXBP1 mutations in early infantile epileptic encephalopathy with suppression-burst pattern. Epilepsia 2010;51:23972405.

14. Milh M, Villeneuve $\mathrm{N}$, Chouchane $\mathrm{M}$, et al. Epileptic and nonepileptic features in patients with early onset epileptic encephalopathy and STXBP1 mutations. Epilepsia 2011;52:1828-1834.

15. Hamdan FF, Piton A, Gauthier J, et al. De novo STXBP1 mutations in mental retardation and nonsyndromic epilepsy. Ann Neurol 2009;65: 748-753.

16. Deprez L, Weckhuysen $S$, Holmgren $P$, et al. Clinical spectrum of early-onset epileptic encephalopathies associated with STXBP1 mutations. Neurology 2010;75:1159-1165.

17. Hamdan FF, Gauthier J, Dobrzeniecka S, et al. Intellectual disability without epilepsy associated with STXBP1 disruption. Eur J Hum Genet 2011;19: 607-609.

18. Schulze KL, Littleton JT, Salzberg A, et al. rop, a Drosophila homolog of yeast Sec1 and vertebrate $\mathrm{n}-\mathrm{Sec} 1 /$ Munc-18 proteins, is a negative regulator of neurotransmitter release in vivo. Neuron 1994;13:1099-1108.

19. Burkhardt P, Hattendorf DA, Weis WI, Fasshauer D. Munc18a controls SNARE assembly through its interaction with the syntaxin N-peptide. EMBO J 2008;27:923-933.

20. Hata Y, Slaughter CA, Südhof TC. Synaptic vesicle fusion complex contains unc-18 homologue bound to syntaxin. Nature 1993;366:347-351.

21. Saitsu H, Hoshino $H$, Kato $M$, et al. Paternal mosaicism of an STXBP1 mutation in OS. Clin Genet 2011;80:484-488.

22. Gilman SR, lossifov I, Levy D, Ronemus M, Wigler M, Vitkup D. Rare de novo variants associated with autism implicate a large functional network of genes involved in formation and function of synapses. Neuron 2011;70:898-907

23. Pinto D, Pagnamenta AT, Klei L, et al. Functional impact of global rare copy number variation in autism spectrum disorders. Nature 2010; 466:368-372.

24. Schaaf $\mathrm{CP}$, Zoghbi HY. Solving the autism puzzle a few pieces at a time. Neuron 2011;70:806-808.

25. Lupski JR, Belmont JW, Boerwinkle E, Gibbs RA. Clan genomics and the complex architecture of human disease. Cell 2011;147:32-43.
26. Shen HM, Storb U. Activation-induced cytidine deaminase (AID) can target both DNA strands when the DNA is supercoiled. Proc Natl Acad Sci USA 2004;101:12997-13002

27. Kanazawa K, Kumada S, Kato M, Saitsu H, Kurihara E, Matsumoto N. Choreo-ballistic movements in a case carrying a missense mutation in syntaxin binding protein 1 gene. Mov Disord 2010;25:2265-2267.

28. Crutcher MD, Alexander GE. Movement-related neuronal activity selectively coding either direction or muscle pattern in three motor areas of the monkey. J Neurophysiol 1990;64:151-163.

29. Saitsu H, Tohyama J, Kumada T, et al. Dominant-negative mutations in alphaII spectrin cause West syndrome with severe cerebral hypomyelination, spastic quadriplegia, and developmental delay. Am J Hum Genet 2010;86:881-891.

30. Voas MG, Lyons DA, Naylor SG, Arana N, Rasband MN, Talbot WS. alphallspectrin is essential for assembly of the nodes of Ranvier in myelinated axons. Curr Bio/ 2007; 17:562-568.

31. Ogawa $Y$, Rasband MN. The functional organization and assembly of the axon initial segment. Curr Opin Neurobio/ 2008;18:307-313.

32. Shoukier M, Teske U, Weise A, Engel W, Argyriou L. Characterization of five novel large deletions causing hereditary haemorrhagic telangiectasia. Clin Genet 2008;73:320-330.

33. Wooderchak W, Gedge F, McDonald M, et al. Hereditary hemorrhagic telangiectasia: two distinct ENG deletions in one family. Clin Genet 2010;78:484-489.

34. Mei-Zahav M, Letarte M, Faughnan ME, Abdalla SA, Cymerman U, MacLusky IB. Symptomatic children with hereditary hemorrhagic telangiectasia: a pediatric center experience. Arch Pediatr Adolesc Med 2006;160:596-601.

35. Ozelius $L$, Hewett JW, Page $C E$, et al. The early-onset torsion dystonia gene (DYT1) encodes an ATP-binding protein. Nat Genet 1997;17:40-48.

36. Risch N, de Leon D, Ozelius L, et al. Genetic analysis of idiopathic torsion dystonia in Ashkenazi Jews and their recent descent from a small founder population. Nat Genet 1995:9:152-159.

37. Goodchild RE, Kim CE, Dauer WT. Loss of the dystonia-associated protein torsinA selectively disrupts the neuronal nuclear envelope. Neuron 2005;48:923-932.

38. Risch NJ, Bressman SB, Senthil G, Ozelius LJ. Intragenic Cis and Trans modification of genetic susceptibility in DYT1 torsion dystonia. Am J Hum Genet 2007:80:1188-1193.

39. Huang N, Lee I, Marcotte EM, Hurles ME. Characterising and predicting haploinsufficiency in the human genome. PLoS Genet 2010;6:e1001154.

40. Weterman MA, Sorrentino V, Kasher PR, et al. A frameshift mutation in LRSAM1 is responsible for a dominant hereditary polyneuropathy. Hum $\mathrm{Mol}$ Genet 2012;21:358-370 